\title{
Anal Cancer Patients in a Metropolitan Hospital Present Infection with Multiple Oncogenic Viruses
}

\author{
Kenneth O. Simbiri ${ }^{1,4}$, Hem C. Jha ${ }^{2}$, David H. Henry ${ }^{3}$ \& Erle S. Robertson ${ }^{2}$ \\ ${ }^{1}$ Department of Microbiology and Immunology, Upstate Medical University, R2120A, Weiskotten Hall, 750 \\ East Adams Street, Syracuse, NY 13210, USA \\ ${ }^{2}$ Department of Microbiology, Abramson Cancer Center, Tumor Virology Program, Perelman School of \\ Medicine at the University of Pennsylvania, 202A Johnson Pavilion, 3610 Hamilton Walk, Philadelphia, PA \\ 19104-6076, USA \\ ${ }^{3}$ Pennsylvania Hospital, 230 W Washington Square, 2nd Floor Philadelphia, PA 19106, USA \\ ${ }^{4}$ Jaramogi Oginga Odinga University of Science and Technology, Bondo, Kenya \\ Correspondence: Kenneth O. Simbiri, Department of Microbiology and Immunology, Upstate Medical \\ University, R2120A, Weiskotten Hall, 750 East Adams Street, Syracuse, NY 13210, USA. Tel: \\ 315-464-5468. Fax: 315-464-4417. E-mail: simbirik@upstate.edu
}

Received: June 10, 2015 Accepted: July 12, 2015 Online Published: August 32015

doi:10.5539/cco.v4n2p32 URL: http://dx.doi.org/10.5539/cco.v4n2p32

This work was supported by the Public Health awards from the National Cancer Institute of the National Institutes of Health (CA137894, CA138434, CA72150, CA91792, CA91792-S1, CA91792-S2, CA108461 to ESR); and the National Institute of Allergy and Infectious Diseases (A167037 to ESR)

\begin{abstract}
Background: Oncoviruses such as HPV, KSHV, and EBV have been reported in patients with HIV/AIDS. Exactly how oncovirus-associated cancers rise in AIDS patients is not fully established. We highlight results from anal cancer samples that were screened for certain known oncoviruses in HIV/AIDS patients.

Methods: Tissues were obtained from 14 anal cancer patients. DNA was extracted from the tissues and used in PCR assays to identify the oncoviruses. Immunohistochemical assays were performed on slides using specific antibodies.

Results: Using PCR we detected HPV-16 ( $\mathrm{P}<0.0001)$, HPV-45 ( $\mathrm{P}<0.0001)$, HPV-31 (P<0.0001), HPV-33 $(\mathrm{P}<0.0001)$, and EBV $(\mathrm{P}<0.0001)$ which were statistically significantly different, and KSHV $(\mathrm{P}=0.090)$. Utilizing immunohistochemistry, the samples were statistically significantly different in HPV-16 E6 (P<0.0001), HPV-16 E7 ( P<0.0001), EBV ( $\mathrm{P}<0.0001)$, and KSHV $(\mathrm{P}<0.0001)$.

Conclusion: The findings indicate that oncogenic human papilloma and herpesviruses are present in anal cancer tissues. Their causal role to the cancer may have implications for long-term pathogenesis.
\end{abstract}

Keywords: HIV-1, EBV, HPV, KSHV, and Anal cancer, oncogenic virus

\section{Introduction}

It has been reported that greater than $15 \%$ of human cancers are currently associated with viruses that are major contributors to disease (zur Hausen, 1991). The oncogenic viruses possess different genomes, life cycles, and represent a number of virus families. The course from viral infection to tumor initiation is slow and may take years after primary infection. Virus infection is generally not sufficient for cancer initiation, with additional epigenetic events and host factors such as immunosuppression, somatic mutations, and genetic predisposition possibly contributing (Liao, 2006). We have shown association between herpesviruses and human papilloma viruses (HPV) with some of the cancers in our study (Simbiri et al., 2010). Sub-Saharan Africa and most of the developing countries have seen a spike in viral associated cancers in Human immunodeficiency virus (HIV) infected patients (Simbiri et al., 2010).

In this study we looked at the association of human papilloma and herpesviruses in anal cancer among 
HIV-infected patients. Anal cancer is considered to be a non-AIDS defining cancer (NADC). It has been shown to be similar to cervical cancer, which is strongly associated with HPV infection and the presence of pre-cancerous lesions (Bernal et al., 2003; Cooley et al., 2003). The incidence in the HIV positive individuals is as high as 60 fold compared to the HIV negative individuals (Feigal, 2001; Frisch et al., 2001; Rabkin, 2001). An increased incidence of HPV-associated cancers has been observed among AIDS patients with increased elevated immunosuppression, likely a factor of prolonged survival that leads to enhanced risk of some HPV-associated cancers (Chaturvedi et al., 2009). Meanwhile, HPV-associated anal cancers in HIV-positive men increased in incidence despite the introduction of highly active antiretroviral therapy (HAART) (Kreuter \& Wieland, 2009). Additionally, in a study of HIV, Epstein Barr virus (EBV), and Kaposi sarcoma herpes virus (KSHV) cooperation in the pathogenesis of human malignancies, a decrease of Kaposi's sarcoma (KS), non-Hodgkin's lymphoma (NHL), and primary central nervous system lymphoma (PCNSL) was noted, while Burkitt's lymphoma and Hodgkin's lymphoma decreased slightly under HAART (da Silva et al., 2011). Some studies have suggested that these NADCs have not become more prevalent (Romassi \& Nagle, 2008; Silverberg $\&$ Abrams, 2007). However, it is evident that these cancers are increasing and are expected to rise in the coming years in the HIV population. The AIDS defining cancers (ADC) are mostly referred to as Kaposi's sarcoma, non-Hodgkin's Lymphoma and cervical cancer. The impact of HAART on the incidence of ADC and NADC among HIV/AIDS patients showed that the overall risk increased in NADCs after introduction of HAART, while the incidence of ADC decreased, and that the increased incidence of NADCs after early use of HAART was likely due to better control of viral replication, increased immunity, and prolonged survival (Cobucci et al., 2014; Pinzone et al., 2012; Kan et al., 2014).

The aim of this study was to identify the oncogenic human papilloma and herpesviruses in anal cancer tissues from patients in Pennsylvania Hospital, Philadelphia to show that oncogenic viruses may be involved in the pathogenesis of the cancer.

\section{Materials and Methods}

We identified 14 anal cancer tissue samples from Pennsylvania Hospital in Philadelphia, Pennsylvania, USA. University of Pennsylvania IRB approved the study (protocol \# 808695). The study was conducted in compliance with the ethical principles of the Declaration of Helsinki. Patients included in the study were adults (age $>18$ years) with histologically proven anal carcinoma malignancy. The patients or their family members signed informed consent document (Table 1).

Table 1. Anal Cancer patient characteristics

\begin{tabular}{clccc}
\hline Case\# & Diagnosis & Age & HIV & HCB/C \\
\hline 1 & Anal carcinoma & - & No & \\
2 & Anal carcinoma-invasive squamous carcinoma & 47 & Yes & \\
3 & Infiltrating moderately differentiated squamous carcinoma & - & No & \\
4 & Invasive squamous cell carcinoma -rectal abscess & 37 & No & \\
5 & Anal carcinoma & 72 & Yes & \\
6 & Squamous cell carcinoma-anal & - & No & \\
7 & Anal intraepithelial neoplasm & 45 & Yes & Hep B \\
8 & Anal cancer & 54 & Yes & \\
9 & Anal cancer & 47 & No & \\
10 & Anal carcinoma & 53 & Yes & \\
11 & Kaposi sarcoma-anal carcinoma & 29 & Yes & \\
12 & Kaposi sarcoma-anal carcinoma & 44 & Yes \\
13 & Kaposi sarcoma-anal carcinoma & - & No \\
14 & Hodgkin's lymphoma-anal carcinoma & - & No & \\
\hline
\end{tabular}

HIV status- (+) vs. (-), p=0.19793, odds ratio- $1.3059,95 \% \mathrm{CI}=0.0254-67.1672$.

Table 1 shows diagnosis, age, HIV status, and hepatitis status of each patient in the study. 


\subsection{DNA Extraction}

Total cellular DNA was extracted from anal tissues by digestion with proteinase $\mathrm{K}$ and phenol-chloroform extraction as previously described (Simbiri et al, 2010). Briefly, tissue samples were prepared by cutting $5 \mu \mathrm{m}$ thick sections using clean blades, followed by de-paraffinization and extraction of the DNA. PCR analysis was performed as previously described (Simbiri et al, 2010) with each virus specific primer including HPV types using consensus primer GP5+/GP6 + that detects more than 20 HPV high-risk (HR) and low- risk (LR) types, as well as EBV, KSHV, JC, and BK viruses.

\subsection{Polymerase Chain Reaction}

The PCR assay was performed with primers as indicated in Table 2. The PCR conditions were as follows: HPVGP5+/GP6+ $94^{\circ} \mathrm{C}-5$ minutes, $94^{\circ} \mathrm{C}-30$ seconds, $48^{\circ} \mathrm{C}-30$ seconds, and $72^{\circ} \mathrm{C}-30$ seconds, for 40 cycles; elongation at $72^{\circ} \mathrm{C}-5$ minutes and incubation at $4^{\circ} \mathrm{C}$. EBV- $94^{\circ} \mathrm{C}-5$ minutes, $94^{\circ} \mathrm{C}-30$ seconds, $47^{\circ} \mathrm{C}-30$ seconds, and $72^{\circ} \mathrm{C}-30$ seconds, for 40 cycles; elongation at $72^{\circ} \mathrm{C}-5$ minutes and incubation at $4^{\circ} \mathrm{C}$. KSHV$94^{\circ} \mathrm{C}-5$ minutes, $94^{\circ} \mathrm{C}-30$ seconds, $52^{\circ} \mathrm{C}-30$ seconds, and $72^{\circ} \mathrm{C}-30$ seconds, for 40 cycles; elongation at $72^{\circ} \mathrm{C}-5$ minutes and incubation at $4^{\circ} \mathrm{C}$. JC- $94^{\circ}-5$ minutes, $94^{\circ}-10$ seconds, $58^{\circ} \mathrm{C}-5$ seconds, $72^{\circ} \mathrm{C}-10$ seconds, for 35 cycles; elongation at $72^{\circ} \mathrm{C}-5$ minutes and incubation at $4^{\circ} \mathrm{C}$; and for $\mathrm{BK}-94^{\circ} \mathrm{C}-5$ minutes, $94^{\circ} \mathrm{C}-25$ seconds, $45^{\circ} \mathrm{C}-10$ seconds, $72^{\circ} \mathrm{C}-25$ seconds, for 35 cycles; elongation at $72^{\circ} \mathrm{C}-5$ minutes and incubation at $4^{\circ} \mathrm{C}$. Annealing temperatures for specific HPV primers were adjusted accordingly. The PCR products were analyzed on $2.5 \%$ agarose gel at $100 \mathrm{~V}$ for 1 hour.

Table 2. Primers used in PCR analysis.

\begin{tabular}{lll}
\hline Primer & Sequence & Size (bp) \\
\hline HPV-L1 & 5'-TTTGTTACTGTGGTAGATACTAC-3' & 150 \\
& 3'-CTTATACTAAATGTCAAATAAAAAG-5' & \\
EBV-BamH1W & 5'-CCAGACAGCAGCCAATTGTC-3' & 129 \\
& 3'-GGTAGAAGACCCCCTCTTAC-5' & \\
KSHV-ORF-73 & 5'-CCATCTCTTGCATTGCCAC-3', & 293 \\
& 5'-AACTACGGTTGGCGAAGTCA-3' & \\
JC VP2/VP3 & 5'-GAAGAACCCAAAACTATTTGTTGAAA-3', & 133 \\
& 5'-GCCTAACTGGAGACAATCTAGAATAATAGTC-3' & \\
BK- Large T & 5'-AAATCCATTTTATCTAATATATG-3, & 370 \\
& 5'-CATATATTAGATAAAATGGATTT-3' & \\
\hline
\end{tabular}

Table 2 shows the primers, primer sequences, and product sizes in the PCR analysis.

\subsection{Immunohistochemistry}

Immunohistochemistry was performed as previously reported (Simbiri et al., 2010). Briefly, we used commercial antibodies HPV-16E6, HPV-16E7, and HPV-16E6/E7 (DAKO Inc., Carpentaria, CA), and monoclonal antibody S12 for EBV-LMP1, and monoclonal antibody derived from KSHV encoded LANA on $5 \mu \mathrm{m}$ slides as previously reported (Simbiri et al., 2010).

\subsection{Data Analysis}

The data for this study was collected in Excel and imported into a statistical software program. Analysis encompassed determination of proportions (MedCalc Software bvba MedCalc Software, Ostend, Belgium).

\section{Results}

We identified the oncogenic human papilloma and herpesviruses in the tissues derived from anal cancer. In this study using PCR and immunohistochemistry we identified human papilloma and herpesviruses in tissue samples from anal cancer patients (Table 3). 
Table 3. Oncogenic viruses identified by PCR in anal cancer tissues

\begin{tabular}{ccccccccccc}
\hline & EBV & HPV-G5+/6+ & HPV-18 & HPV-16 & HPV-45 & HPV-11 & HPV-31 & HPV-33 & KSHV \\
\hline 1 & + & - & + & - & + & - & + & - & - & + \\
2 & + & + & + & + & - & + & + & + & + \\
3 & + & + & + & + & + & - & + & - & + \\
4 & + & - & - & + & - & + & - & - & - \\
5 & + & - & - & + & - & - & - & - & - \\
6 & + & + & + & + & - & + & + & + & + \\
7 & + & + & - & + & - & - & + & - & - \\
8 & + & + & + & + & + & - & + & - & + \\
9 & + & - & - & + & - & + & + & - & - \\
10 & + & + & + & + & - & + & + & - & - \\
11 & + & - & - & + & + & - & + & + & - \\
12 & + & + & + & + & - & + & + & - & + \\
13 & + & + & - & + & - & + & - & - & - \\
14 & + & - & - & + & - & - & - & - & - \\
Total & 14 & 8 & 6 & 14 & 3 & 8 & 9 & 3 & 6 \\
Percent & $100 \%$ & $56 \%$ & $44 \%$ & $100 \%$ & $22 \%$ & $56 \%$ & $67 \%$ & $22 \%$ & $44 \%$ \\
OR & 40401 & 1.6198 & 0.6173 & 40401 & 0.0796 & 1.6198 & 4.1221 & 0.0796 & 0.6173 \\
CI & $79-20651$ & $0.92-2.83$ & $0.35-1.07$ & $793-20651$ & $0.04-0.15$ & $0.92-2.82$ & $2.28-7.46$ & $0.04-0.15$ & $0.35-1.07$ \\
P & $<0.0001$ & 0.0905 & 0.0905 & $<0.0001$ & $<0.0001$ & 0.0905 & $<0.0001$ & $<0.0001$ & 0.0905 \\
\hline
\end{tabular}

Table 3 shows oncogenic viruses identified in each anal cancer patient by PCR analysis. Most cases tested positive for HPV types, EBV, and KSHV.

Utilizing PCR we detected HPV using GP5+/GP6+ in $56 \%$ of the samples $(\mathrm{P}=0.091$, OR 1.62 , 95\% CI 0.92-2.83); HPV-18 in 44\% ( $\mathrm{P}=0.091$, OR 0.62, 95\% CI 0.35-1.07); HPV-16 in $100 \%$ (p<0.0001, OR 40401, $95 \%$ CI 793-20651); HPV-45 in 22\% ( $\mathrm{P}<0.0001$, OR 0.08, 95\% CI 0.04-0.15); HPV-11 in 56\% ( $\mathrm{P}=0.09, \mathrm{OR}$ 1.62, 95\% CI 0.92-2.82); HPV-31 in 67\% ( $\mathrm{P}<0.0001$, OR 4.12, 95\% CI2.28-7.46); HPV-33 in $22 \%$ ( $\mathrm{P}<0.0001$, OR 0.80, 95\% CI 0.04-0.15); EBV in $100 \%$ ( $\mathrm{P}<0.0001$, OR 40401, 95\% CI 793-20651); and KSHV in $44 \%$ $(\mathrm{P}=0.09$, OR 0.62, 95\% CI 0.35-1.07) (Table 3 and Figure 1).

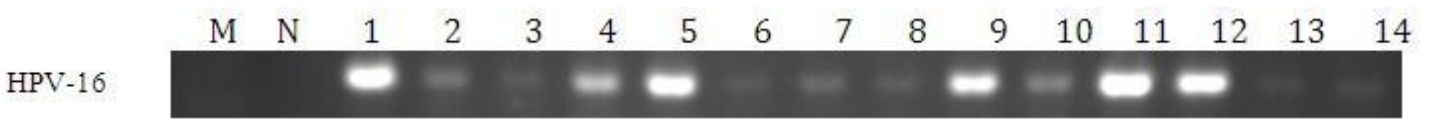

$150 \mathrm{bp}$

Figure 1. Representative HPV-16 PCR for Anal cancer patients

Figure 1 shows a representative PCR result of anal samples processed using HPV-L1 primer. We observed that all the samples contained HPV genome. M-marker; $\mathrm{N}-\mathrm{H}_{2} \mathrm{O}$ negative control; Samples, 1-14.

Further, when we used immunohistochemistry, we detected HPV-16 E6 in 88.9\% (P<0.0001, OR 65.46, 95\% CI 26.9-158.7); HPV-16 E7 was similar to HPV-16 E6 88.9\% (P<0.0001, OR 65.46, 95\%CI 26.9-158.7), and HPV-16 E6/E7 in 100\% (P<0.0001, OR 40401, 95\% CI 793-20651). For EBV we detected 100\% (P<0.0001, OR 40401, 95\% CI 793-20651), and for KSHV we detected 77.8\% (P<0.0001, OR 12.57, 95\% CI 6.4-12.57) (Table 4 and Figure 2). 


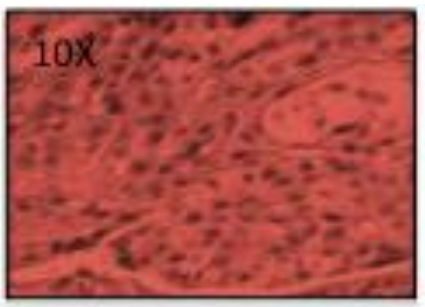

HPV-H\&E

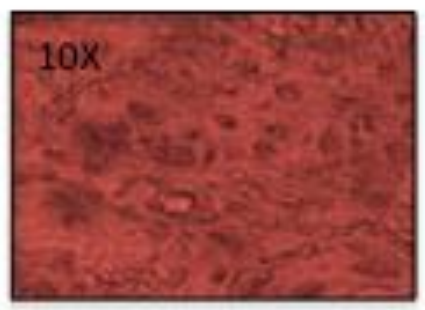

HPV-16 E7

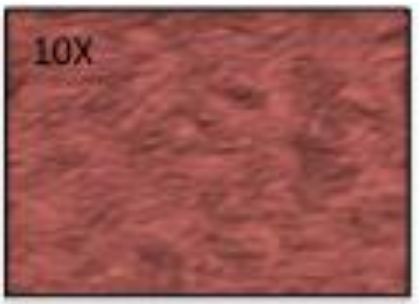

HPV16: Negative control

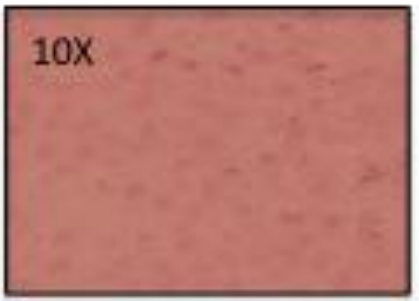

HPV-16 E6/E7

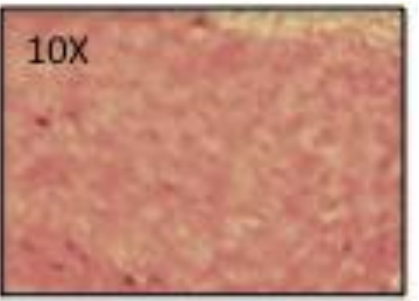

HPV-16 E6

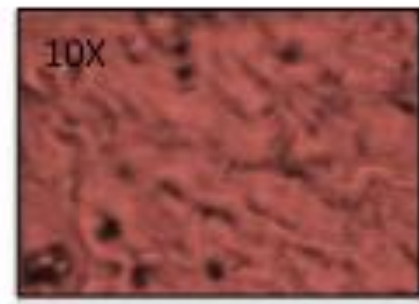

KSHV

Figure 2. Representative Immunohistochemistry of oncoviral proteins detected in anal cancer samples

Figure 2 shows the immunohistochemistry results using specific antibodies. Positive cells stained dark brown. Some of the tissues did not stain robustly possibly due to status of the tissues.

Table 4. Oncogenic viruses identified by Immunohistochemistry in anal cancer tissues

\begin{tabular}{cccccc}
\hline Number of cases & EBV-S12 & LANA & HPV-E6 & HPV-E7 & HPV-E6/E7 \\
\hline 1 & ++ & - & + & - & ++ \\
2 & + & ++ & + & + & +++ \\
3 & + & + & - & ++ & ++ \\
4 & +++ & + & + & + & ++ \\
5 & ++ & + & ++ & + & ++ \\
6 & +++ & ++ & ++ & ++ & ++ \\
7 & + & - & + & +++ & +++ \\
8 & ++ & + & + & +++ & + \\
9 & +++ & + & ++ & ++ & ++ \\
10 & ++ & + & ++ & ++ & ++ \\
11 & ++ & ++ & +++ & ++ & + \\
12 & + & ++ & ++ & ++ & ++ \\
13 & +++ & + & ++ & ++ & ++ \\
14 & ++ & - & - & - & 14 \\
Total & 14 & 11 & 12 & $88.9 \%$ & $100 \%$ \\
Percent & $100 \%$ & $77.8 \%$ & $88.9 \%$ & 65.46 & 40401 \\
OR & 40401 & 12.57 & 65.46 & $26.9-158.7$ & $793-20651$ \\
CI & $793-20561$ & $6.4-12.57$ & $26.9-158.7$ & $<0.0001$ & $<0.0001$ \\
P & $<0.0001$ & $<0.0001$ & $<0.0001$ & & ++
\end{tabular}

Table 4 shows oncogenic viruses identified in each anal cancer patient as determined by IHC analysis. Most cases tested positive for EBV, KSHV, HPV-E6, and HPV-E6/E7. 
High risk, low risk human papilloma viruses and herpesviruses were detected in anal cancer samples retrieved from a local hospital. We were interested in determining if similar oncogenic viruses that we had previously identified in samples from Botswana could be present in anal cancer samples. Thus, we screened samples from HIV patients from Pennsylvania hospital in Philadelphia, a local hospital, to determine if similarity in co-infection persisted. It was interesting to observe that the oncogenic viruses that we had previously identified in Botswana-based samples were also present in the few samples from the region, an indication that these viruses are prevalent, may be associated with the cancer, and may contribute to its pathogenesis through similar pathways. We observed significant differences for EBV, all HPV types except HPV-18, and no significant difference for KSHV using PCR (Table 3). However, on IHC there were significant differences for EBV, KSHV, HPV-16 E6 and E6/E7 (Table 4).

High-risk HPV types were detected in both HIV positive and negative samples. In all anal cancer samples we identified high risk HPV. Other HPV types were also identified (Table 3). These results suggest a high incidence of HPV in patients. The HPV may become opportunistic and oncogenic with long latency period once the patients are immunocompromised due to HIV.

EBV was consistently detected in anal cancer. As has been reported in previous studies (Simbiri et al, 2010), the ubiquitous EBV was again detected to a high degree in all the anal cancer samples (Table 3). In future studies, it will be interesting to identify the EBV type (EBV1 or EBV2) associated with the cancer, or if both EBV types co-infect and may contribute at different levels to pathogenesis.

The cancer was identified in young HIV infected patients. One recurring observation was the detection of the oncogenic viruses and anal cancer in younger HIV infected patients. This may be due to immunosuppression by HIV infection and other opportunistic infections at an early age, which renders their immune system to mount surveillance at low levels similar to that of older individuals who are more susceptible to the infections and associated diseases. The impact of age on the cancer patients with HIV warrants further investigation (Table 4).

\section{Discussion}

In this study we have shown the presence of oncogenic viruses in anal cancer tissues. We show the increase in incidence of the cancer in young HIV-infected patients, and the prevalence of the oncogenic human papilloma and herpesviruses in the cancer.

Our past study detected multiple tumor viruses in OSSN tissues. For the first time we showed the presence of HPV, EBV, KSHV, and CMV in ocular tissues in HIV/AIDS patients (Simbiri et al., 2010), vulvar cancer (Simbiri et al., 2014). Associations of viruses with human cancers have already been reported, such as KSHV with KS and PEL (Kiire \& Dhillon, 2006); EBV with primary CNS lymphoma (Kestelyn et al., 1990), nasopharyngeal carcinoma (Cook-Mozaffari et al, 1998) and Burkitt's lymphoma (Cohen, 2000); HPV with cervical cancer (Mirzamani et al., 2006), anal cancer (Salit et al., 2009), head and neck (Goldenberg et al., 2001), and testicular cancer (Munoz et al., 2003). However, the contribution by HIV to the development of the malignancies remains unclear and may be through a number of different mechanisms that target variable cellular processes.

Viral proteins expressed in infected cells mediate infection and establishment of latency. Importantly, the immunomodulatory mechanisms used by one virus may actually enhance or benefit the activity or replication of other viruses. Many of these viruses (for example, HPV, CMV, and EBV) are quite common in the human population (Waddell et al., 1996) but do not always lead to development of malignancies in healthy individuals. The localization of these oncogenic proteins in ocular surface squamous neoplasia (OSSN) (Simbiri et al., 2010), vulvar cancer (Simbiri et al., 2014), and anal cancer tissues in our present study show association, however establishment of a causal effect by any of the viruses, or a multiple of these viruses in an HIV environment has yet to be fully confirmed. Determining the pathways involved in initiation and establishment of the cancers in HIV patients compared to non-HIV patients will give us a better understanding of the interplay of the different oncogenic viral proteins in the normal immune state and immunosuppressed state.

The localization of these tumor viruses in the different cancer tissue sections suggest that these viruses may functionally interact to contribute to OSSN, vulvar, and anal cancer phenotypes. Similar to our findings of HPV and EBV in ocular, vulvar, and anal tissues, Prayitno reported that in 19 cervical carcinoma samples $89 \%$ were positive for HPV and 68\% were positive for EBV (Prayitno, 2006). It has been suggested that HPV probably does not act alone in initiating ocular neoplasia, but that other factors including the ultraviolet light are involved (Nakamura et al., 1997). Previous reports have suggested that infection with more than one HPV type enhances cervical cancer and oral squamous carcinoma (Premoli-De-Percoco et al., 1998). Mirzamani et al. reported co-infections with EBV and HPV-16/18 in nasopharyngeal carcinoma (NPC) patients and concluded that both 
viruses were important in contributing to the pathogenesis of NPC (Mirzamani et al., 2006). The selection pressure that may exist as a consequence of competition between opportunistic infections may further lead to mutations occurring in the HPV types as well as in the other infectious agents that may contribute to oncogenesis.

Our findings in this study showed the presence of similar human papilloma and herpesviruses in the anal samples examined as was observed on OSSN and vulvar samples. HAART has been shown not to alter HPV persistence or rates of progression or regression of premalignant anogenital lesion (Salit et al., 2009; Palefsky et al., 2005; Heard et al., 2004). Palesfky et al. suggest that the increase in anal cancer in the HAART era may reflect the likelihood that in the pre-HAART era patients at highest risk of anal cancer would have died from other causes (Palefsky et al., 2005; Heard et al., 2004). Prior to the introduction of large scale HAART therapy in Botswana in 2002 (WHO, 2006) and other regions, most of the patients who had these oncogenic neoplasias pre-HAART era died without diagnosis or therapy. In future a longitudinal study that addresses the impact of HAART on anal cancer in relation to associated oncogenic viruses need to be initiated. Equally significant would be to investigate the effect of HIV on selection of other infections such as bacteria microbiome that may affect pathogenesis as was shown in our previous findings (Simbiri \& Robertson, 2012).

Though this study had a small sample size it highlights the significance of human papilloma and herpesviruses in the pathogenesis of this cancer and other cancers in HIV/AIDS patients that will lead to new therapies and prevention strategies.

\section{Competing interests}

The authors declare that they have no competing interests.

\section{Authors' contributions}

KOS designed the study, conducted experiments, interpreted data, drafted the manuscript and supervised the study, HCJ interpreted data and drafted the manuscript, DHH identified anal cancer tissues and drafted the manuscript, and ESR designed the study, interpreted data, edited the manuscript, and supervised the study. All authors have read and approved the final manuscript.

\section{Acknowledgements}

The authors thank Jacqueline O'Duor who collected patient samples and pertinent data from Pennsylvania Hospital. We would also like to extend special thanks to all the patients and physicians as well as the personnel at the Pennsylvania Hospital without whom this study would have been possible.

\section{References}

Bernal, M., Gomez, F. J., \& Gomez, G. J. (2003). Cancer and AIDS. Eur J Cancer Prev, 12, 171-2.

Chaturvedi, A. K., Madeleine, M. M., Biggar, R. J., \& Engels, E. A. (2009). Risk of human papillomavirus-associated cancers among persons with AIDS. J Natl Cancer Inst., 101(16), 1120-30. http://dx.doi.org/10.1093/jnci/djp205

Cobucci, R. N., Lima, P. H., de Souza, P. C., Costa, V. V., Cornetta, M. D., Fernandes, J. V., \& Gonçalves, A. K. (2014). Assessing the impact of HAART on the incidence of defining and non-defining AIDS cancers among patients with HIV/AIDS: A systematic review. J Infect Public Health, pii: S1876-0341(14)00141-5. http://dx.doi.org/10.1016/j.jiph.2014.08.003

Cohen JI. (2000). Epstein-Barr virus infection. $N$ Engl $J$ Med., 343, 481-492. http://dx.doi.org/10.1056/NEJM200008173430707

Cook-Mozaffari, P., Newton, R., Beral, V., \& Burkitt, D. P. (1998). The geographical distribution of Kaposi's sarcoma and of lymphomas in Africa before the AIDS epidemic. Br J Cancer, 78, 1521-1528. PMID: 9836488.

Cooley, T. P. (2003). Non-AIDS-defining cancer in HIV-infected people. Hematol Oncol Clin North Am, 17, 889-99. PMID: 12852661.

da Silva, S. R, \& de Oliveira, D. E. (2011). HIV, EBV and KSHV: viral cooperation in the pathogenesis of human malignancies. Cancer Lett., 305(2),175-85. http://dx.doi.org/10.1016/j.canlet.2011.02.007

Feigal, E. G. (2001). AIDS malignancy clinical research: resources of the National Cancer Institute. Cancer Treat Res, 104, 367-72. PMID: 11191135.

Frisch, M., Biggar, R. J., Engels, E. A., \& Goedert, J. J. (2001). Association of cancer with AIDS-related immunosuppression in adults. Jama, 285, 1736-45. PMID: 11277828 
Goldenberg, D., Golz, A., Netzer, A., Rosenblatt, E., Rachmiel, A., Goldenberg, R. F., \& Joachims, H. Z. (2001). Epstein-Barr virus and cancers of the head and neck. Am J Otolaryngol., 22, 197-205. http://dx.doi.org/10.1053/ajot.2001.23429

Heard, I., Palefsky, J. M., \& Kazatchkine, M. D. (2004). The impact of HIV antiviral therapy on human papillomavirus (HPV) infections and HPV-related diseases. Antiviral therapy, 9(1), 13-22. PMID: 15040532.

John, B. L. (2006). Viruses and Human Cancer. Yale J Biol Med., 79(3-4), 115-122. PMCID: PMC1994798.

Kan, M., Wong, P. H. P., Press, N., \& Wiseman, S. M. (2014). Colorectal and anal cancer in HIV/AIDS patients: a comprehensive review. Expert review of anticancer therapy, 14(4), 395-405. http://dx.doi.org/10.1586/14737140.2013.877843

Simbiri, K. O., \& Robertson, E. S. (2012). Characterization of the OSSN microbiome in HIV-1 infected patients. Cancer and Clinical Oncology, 1(1), p88. http://dx.doi.org/10.5539/cco.v1n1p88

Kestelyn, P. H., Stevens, A. M., Ndayambaje, A., Hanssens, M., \& Van De Perre, P. H. (1990). HIV and conjunctival malignancies. The Lancet, 336(8706), 51-52. http://dx.doi.org/10.1016/0140-6736(90)91562-O

Kiire, C. A., \& Dhillon, B. (2006). The aetiology and associations of conjunctival intraepithelial neoplasia. British journal of ophthalmology, 90(1), 109-113. http://dx.doi.org/10.1136/bjo.2005.077305

Kreuter, A., \& Wieland, U. (2009). Human papillomavirus-associated diseases in HIV-infected men who have sex with men. Current opinion in infectious diseases, 22(2), 109-114. http://dx.doi.org/10.1097/QCO.0b013e3283229fc8

Mirzamani, N., Salehian, P., \& Farhadi, M. (2006). Detection of EBV and HPV in nasopharyngeal carcinoma by in situ hybridization. Experimental and molecular pathology, 81(3), 231-234. http://dx.doi.org/10.1016/j.yexmp.2006.04.006

Nakamura, Y., Mashima, Y., Kameyama, K., Mukai, M., \& Oguchi, Y. (1997). Detection of human papillomavirus infection in squamous tumours of the conjunctiva and lacrimal sac by immunohistochemistry, in situ hybridisation, and polymerase chain reaction. British journal of ophthalmology, 81(4), 308-313. http://dx.doi.org/10.1136/bjo.81.4.308

Palefsky, J. M., Holly, E. A., Efirdc, J. T., Da Costa, M., Jay, N., Berry, J. M., \& Darragh, T. M. (2005). Anal intraepithelial neoplasia in the highly active antiretroviral therapy era among HIV-positive men who have sex with men. Aids, 19(13), 1407-1414. PMID: 16103772.

Pande, S., Jain, N., Prusty, B. K., Bhambhani, S., Gupta, S., Sharma, R., ... Das, B. C. (2008). Human papillomavirus type 16 variant analysis of E6, E7, and L1 genes and long control region in biopsy samples from cervical cancer patients in north India. Journal of clinical microbiology, 46(3), 1060-1066. http://dx.doi.org/10.1128/JCM.02202-07

Pinzone, M. R., Fiorica, F., Di Rosa, M., Malaguarnera, G., Malaguarnera, L., Cacopardo, B., ... Nunnari, G. (2012). Non-AIDS-defining cancers among HIV-infected people. Eur Rev Med Pharmacol Sci, 16(10), 1377-1388. PMID: 23104654.

Prayitno, A. (2006). Cervical cancer with human papilloma virus and Epstein Barr virus positive. Journal of carcinogenesis, 5(1), 13. http://dx.doi.org/10.1186/1477-3163-5-13

Premoli-De-Percoco, G., Ramirez, J. L., \& Galindo, I. (1998). Correlation between HPV types associated with oral squamous cell carcinoma and cervicovaginal cytology: an in situ hybridization study. Oral Surgery, Oral Medicine, Oral Pathology, Oral Radiology, and Endodontology, 86(1), 77-81. http://dx.doi.org/10.1016/S1079-2104(98)90153-6

Rabkin, C. S. (2001). AIDS and cancer in the era of highly active antiretroviral therapy (HAART). European Journal of Cancer, 37(10), 1316-1319. PMID: 11423263.

Romassi, M., \& Nagle, D. (2008). Images in HIV/AIDS. The changing face of anal cancer. The AIDS reader, 18(4), 185. PMID: 18472440.

Salit, I. E., Tinmouth, J., Chong, S., Raboud, J., Diong, C., Su, D., ... Mahony, J. (2009). Screening for HIV-associated anal cancer: correlation of HPV genotypes, p16, and E6 transcripts with anal pathology. Cancer Epidemiology Biomarkers \& Prevention, 18(7), 1986-1992. http://dx.doi.org/10.1158/1055-9965.EPI-08-1141

Silverberg, M. J., \& Abrams, D. I. (2007). AIDS-defining and non-AIDS-defining malignancies: cancer occurrence in the antiretroviral therapy era. Current opinion in oncology, 19(5), 446-451. PMID: 17762569. 
Simbiri, K. O., Jha, H. C., Kayembe, M. K., Kovarik, C., \& Robertson, E. S. (2014). Oncogenic viruses associated with vulva cancer in HIV-1 patients in Botswana. Infectious agents and cancer, 9(1), 28. http://dx.doi.org/10.1186/1750-9378-9-28

Simbiri, K. O., Murakami, M., Feldman, M., Steenhoff, A. P., Nkomazana, O., Bisson, G., \& Robertson, E. S. (2010). Multiple oncogenic viruses identified in Ocular surface squamous neoplasia in HIV-1 patients. Infect Agent Cancer, 5(6), 1750-93. http://dx.doi.org/10.1186/1750-9378-5-6

Waddell, K. M., Downing, R. G., Lucas, S. B., \& Newton, R. (2006). Corneo-conjunctival carcinoma in Uganda. Eye, 20(8), 893-899. http://dx.doi.org/10.1038/sj.eye.6702043

Waddell, K. M., Lewallen, S., Lucas, S. B., Atenyi-Agaba, C., Herrington, C. S., \& Liomba, G. (1996). Carcinoma of the conjunctiva and HIV infection in Uganda and Malawi. British Journal of Ophthalmology, 80(6), 503-508. http://dx.doi.org/10.1136/bjo.80.6.503

Zur Hausen, H. (1991). Viruses in human cancers. Science, 254(5035), 1167-1173.

\section{Copyrights}

Copyright for this article is retained by the author(s), with first publication rights granted to the journal.

This is an open-access article distributed under the terms and conditions of the Creative Commons Attribution license (http://creativecommons.org/licenses/by/3.0/). 